\title{
MCSD: A MATLAB Tool for Monte-Carlo Simulations of Diffusion in biological Tissues
}

David N. Sousa ${ }^{1}$ and Hugo A. Ferreira ${ }^{2}$

1 William James Center for Research, ISPA - Instituto Universitário 2 Instituto de Biofísica e Engenharia Biomédica, Faculdade de Ciências da Universidade de Lisboa

DOI: $10.21105 /$ joss.00966

\section{Software}

- Review ¿

- Repository ๘

- Archive ${ }^{\pi}$

Submitted: 09 August 2018

Published: 26 October 2018

\section{License}

Authors of papers retain copyright and release the work under a Creative Commons Attribution 4.0 International License (CC-BY).

\section{Summary} open-source MATLAB tool developed for that purpose.
Understanding diffusion processes is particularly important for clinical imaging to distinguish between healthy and pathological tissues or between benign and malignant lesions. Computational models of diffusion allow us to predict specific architectural properties of biological tissues which correlate to the measured values of diffusion imaging, thus providing a deeper understanding of certain pathologies in vivo. Here we present a free and

In homogeneous and isotropic media, diffusion has a Gaussian behavior and is statistically described by Einstein's Brownian motion equation. A probability density function (PDF) of the proportion of particles displaced in a given direction (Einstein, 1956). In complex heterogeneous environments such as biological tissues, the movement of water molecules is highly constrained by barriers such as those related to cellular components: membranes, organelles and large proteins, in which case, diffusion has a non-gaussian behavior, and tissue micro-structural complexity is characterized by observing the deviation from a normal distribution (Jensen \& Helpern, 2010).

Diffusion-Weighted Imaging (DWI), Diffusion Kurtosis Imaging (DKI) and Diffusion Tensor Imaging (DTI), are different Magnetic Resonance Imaging (MRI) techniques which take advantage of water diffusion properties in biological tissues for the purpose of characterizing tissue micro-structural complexity in vivo. These techniques have shown to be very useful in quantifying the complexity of tissue barriers, and for characterizing microstructural changes due to injury, treatment, disease or even normal physiological changes such as aging, including in cases of anisotropic diffusion, namely the case of white matter in the brain, where diffusion is essentially parallel to axonal membranes but highly restricted in the directions perpendicular to axons (Basser, Mattiello, \& LeBihan, 1994a, 1994b; Fieremans, Jensen, \& Helpern, 2011; Henriques, Correia, Nunes, \& Ferreira, 2015; Jensen, Helpern, Ramani, Lu, \& Kaczynski, 2005).

Monte-Carlo simulations can be used to investigate the sensitivity of models of diffusion, kurtosis of diffusion, and anisotropy and further offer a deeper understanding on the various parameters defining the complexity of biological tissues, such as the cell radii distribution and type, permeability of membranes, intracellular volume fraction and values of intracellular and extracellular diffusion. (Fieremans, Novikov, Jensen, \& Helpern, 2010; Lee, Bennett, \& Debbins, 2013; Meier, Dreher, \& Leibfritz, 2003; Sousa \& Ferreira, 2015; Szafer, Zhong, \& Gore, 1995). By correlating theoretical and computational models insights with knowledge acquired from imaging techniques it is possible to better understand various pathologies.

Some theoretical and computational models were developed in this direction, but as far as we know only one free open-source tool, the UCL Camino Diffusion MRI Toolkit (Cook et 
al., 2006), was designed and made available for researchers in this field to test their basic predictions. The Monte-Carlo Simulations of Diffusion MCSD package and the tutorial we present here were created for that purpose and their aim is to be simple and useful for both beginners and advanced users of MATLAB.

MCSD is a simple and free open-source MATLAB (The MathWorks Inc, 2018) tool designed to simulate diffusion processes in complex environments such as biological tissues. It allows to investigate the sensitivity of models of diffusion, kurtosis of diffusion, and anisotropy, and it can be used to complement our knowledge about microstructural changes due to injury, treatment, disease or even normal physiological changes such as aging. Currently, MCSD is used by the the Brain Connectivity Project (http://hatdferreira.wixsite.com/brainconnectivity/home) to investigate such problems. It is very useful for researchers in medicine, neuroscience and biomedical engineering, and because of its flexibility, this package might also be useful for researchers from other fields.

With this tool it is possible to create 1, 2 or 3 -D cell environments and simulate the brownian motion of particles subject to movement constraints, such as permeable or impermeable cell membranes or other types of barriers the user may want to define when designing his own complex environment. Additionally, MCSD offers a small set of functions to calculate particles' displacements, and any measures the user may want to define, as well as their compartmental components in all cartesian directions.

\section{Installation and details}

The MATLAB toolbox MCSD is available at https://github.com/davidnsousa/mcsd. In MATLAB the Statistics and Machine Learning Toolbox is required for a complete functioning of the package. MCSD is fully compatible with Octave too. To use it download the package and add the folder mcsd/mcsd MATLAB/Octave search path via the addpath() MATLAB/Octave built-in function. MCSD provides the following functions:

- cells() designs 1, 2 and 3-D cell environments according to a user defined cell radii distribution and a specified region to pack the cells.

- rwalkfree() generates the random walk of one or multiple particles in a free environment without any barriers.

- $r w a l k()$ generates the random walk of one or multiple particles in the presence of barriers.

- displacement() calculates the displacement of the particles in every orthogonal direction.

- cmeasures() calculates a user-defined measure of the particles' displacement distribution in all cartesian directions, including compartmental components.

- where() indicates what particles are inside or outside compartments at a specific step of the random walk trajectory.

- fanisotropy() calculates the fractional anisotropy of diffusion.

In the MATLAB/Octave command line type help followed by the name of each one of the functions above for further details about input and output parameters. In the github repository, at tutorial/, the user can also find a tutorial with more details and various examples. A replication script of the tutorial examples is also provided for MATLAB users.

Combining theoretical and computational insights about diffusion processes with the knowledge acquired from imaging techniques has proved to be an important research direction for understanding the micro-structural complexity of biological tissues. But, 
as yet, no simple and free open-source tools are available for researchers in this field to test their basic predictions. MCSD offers such possibility. The functions provided by MCSD are highly flexible and useful for the design of complex random walk environments such as biological tissues. In fact, although MCSD was developed specifically to simulate diffusion processes in such environments, researchers from other fields might find this package useful as well.

Basser, P. J., Mattiello, J., \& LeBihan, D. (1994a). Estimation of the effective selfdiffusion tensor from the nmr spin echo. Journal of Magnetic Resonance, Series B, 103(3), 247-254. doi:10.1006/jmrb.1994.1037

Basser, P. J., Mattiello, J., \& LeBihan, D. (1994b). MR diffusion tensor spectroscopy and imaging. Biophysical journal, 66(1), 259-267. doi:10.1016/S0006-3495(94)80775-1

Cook, P., Bai, Y., Nedjati-Gilani, S., Seunarine, K., Hall, M., Parker, G., \& Alexander, D. C. (2006). Camino: Open-source diffusion-mri reconstruction and processing. In 14th scientific meeting of the international society for magnetic resonance in medicine (Vol. 2759, p. 2759). Seattle WA, USA.

Einstein, A. (1956). Investigations on the theory of the brownian movement. Courier Corporation.

Fieremans, E., Jensen, J. H., \& Helpern, J. A. (2011). White matter characterization with diffusional kurtosis imaging. Neuroimage, 58(1), 177-188. doi:10.1016/j.neuroimage.2011.06.006

Fieremans, E., Novikov, D. S., Jensen, J. H., \& Helpern, J. A. (2010). Monte carlo study of a two-compartment exchange model of diffusion. NMR in Biomedicine, 23(7), 711-724. doi:10.1002/nbm.1577

Henriques, R. N., Correia, M. M., Nunes, R. G., \& Ferreira, H. A. (2015). Exploring the 3D geometry of the diffusion kurtosis tensor-impact on the development of robust tractography procedures and novel biomarkers. Neuroimage, 111, 85-99. doi:10.1016/j.neuroimage.2015.02.004

Jensen, J. H., \& Helpern, J. A. (2010). MRI quantification of non-gaussian water diffusion by kurtosis analysis. NMR in Biomedicine, 23(7), 698-710. doi:10.1002/nbm.1518

Jensen, J. H., Helpern, J. A., Ramani, A., Lu, H., \& Kaczynski, K. (2005). Diffusional kurtosis imaging: The quantification of non-gaussian water diffusion by means of magnetic resonance imaging. Magnetic Resonance in Medicine: An Official Journal of the International Society for Magnetic Resonance in Medicine, 53(6), 1432-1440. doi:10.1002/mrm.20508

Lee, C.-Y., Bennett, K. M., \& Debbins, J. P. (2013). Sensitivities of statistical distribution model and diffusion kurtosis model in varying microstructural environments: A monte carlo study. Journal of Magnetic Resonance, 230, 19-26. doi:10.1016/j.jmr.2013.01.014

Meier, C., Dreher, W., \& Leibfritz, D. (2003). Diffusion in compartmental systems. I. A comparison of an analytical model with simulations. Magnetic Resonance in Medicine: An Official Journal of the International Society for Magnetic Resonance in Medicine, 50(3), 500-509. doi:10.1002/mrm.10557

Sousa, D. N., \& Ferreira, H. A. (2015). Diffusion kurtosis imaging: Monte carlo simulation of diffusion processes using crowdprocess. In Bioengineering (enbeng), 2015 ieee 4th portuguese meeting on (pp. 1-4). IEEE. doi:10.1109/ENBENG.2015.7088857

Szafer, A., Zhong, J., \& Gore, J. C. (1995). Theoretical model for water diffusion in tissues. Magnetic resonance in medicine, 33(5), 697-712. doi:10.1002/mrm.1910330516

The MathWorks Inc. (2018). MATLAB - the language of technical computing, version 9.4 (r2018a). Natick, Massachusetts: The MathWorks, Inc. 